\title{
Ueber Aethylenchlorbromid und Abkömmlinge des Aethylenchlorsulfocyanids;
}

\author{
von \\ Dr. J. W. James.
}

Im Anschluss an meine vor drei Jahren über A ethylenchlorsulfocyanid mitgetheilten Versuche ${ }^{1}$ ) berichte ich im Folgenden über weitere, darauf bezügliche Beobachtungen.

\section{Darstellung des Aethylenchlorbromids nach Maxwell Simpson.}

Kurz nachdem es mir gelungen war, das Aethylenchlorsulfocyanid aus dem nach der Lössner'schen Methode dargestellten Aethylenchlorbromid zu erhalten, schlug Maxwell Simpson eine andere Methode zur Darstellung der letzteren Verbindung vor, mit Hülfe deren (nach Vornahme einiger kleiner Abänderungen) es mir möglich war, bedeutend besser, schneller und leichter zu arbeiten. Vor allem ist die $70 \mathrm{pCt}$. betragende Ausbeute von bei $107^{\circ}-108^{\circ}$ siedendem, reinem Chlorbromid bemerkenswerth.

Da über diese vorzügliche Methode in den verschiedensten chemischen Journalen nur aus den "Proceedings of the Royal Society of London" kurz referirt worden ist, und da durch meine Beobachtung, nach welcher es möglich ist, in dem Aethylenchlorbromid das Brom allein durch ein zusammengesetztes negatives Radical zu ersetzen, die gemischten Haloïdderivate der Aethylenreihe ein neues Interesse gewonnen haben, so erlaube ich mir die Beschreibung der Methode in der Uebersetzung mitzutheilen:

Simpson sagt: „500 "grains" Brom (etwas mehr wie 32 Grm.) werden in 4 "Fluid ounces" einer Mischung von gleichem Volumen starker Salzäure ${ }^{2}$ ) und Wasser gelöst (?). Die Lösung wird in einen Kolben mit langem Hals gebracht,

1) Dies, Journ, [2] 20,351 ff:

2) Sowohl in der Originalabhandlung, als auch in den Referaten wird "Unterchlorige Säure" angegeben, was natürlich ein Druckfehler ist. 
mit Eis umgeben und dann durch Wasser gereinigtes Chlor unter häufigem Schütteln so lange in dieselbe geleitet, bis dieses nicht mehr absorbirt wird. Auf diese Weise geht keine Spur von Brom verloren, noch wird während des Durchleitens des Chlors irgend ein festes Hydrat gebildet. Leitete ich darauf Aethylen in diese häufig durchgeschüttelte und mit kaltem Wasser umgebene Flüssigkeit, so erhielt ich eine grosse Menge eines Oels, welches von der sauren Lösung getrennt, mit Kalilauge gewaschen und dann destillirt wurde. Fast die ganze Menge ging zwischen $106^{\circ}-110^{\circ}$ über, meistens zwischen $108^{\circ}-110^{\circ}$."

Als ich zuerst diese Methode anwandte, um eine grosse Menge des Chlorbromids zu erhalten, brachte ich 500 Grm. Brom in einen passenden, mit Eis umgebenen Kolben mit 30 "Fluid ounces" Salzsäure und dem gleichen Volum Wasser zusammen und leitete nach der von Simpson angegebenen Weise Chlor und Aethylen in die Flüssigkeit. Nach Vollendung der Operation erhielt ich eine grosse Menge eines Oeles, von welchem ein Theil nach dem Waschen und Trocknen von $109^{0}-112^{0}$ überging; ein grösserer Theil jedoch von $112^{\circ}-115^{\circ}$ siedete. Die Ausbente von reinem und unreinem Oel betrug zusammen kaum über 50 pCt., während nur wenige Gramm bei $106^{\circ}-108^{\circ}$ destillirten, dem Siedepunkt des nach der Lössner'schen Methode dargestellten Aethylenchlorbromids. Hierauf liess ich auf das von $109^{\circ}$ $112^{\circ}$ siedende Product Schwefelcyankalium in der unten beschriebenen Weise einwirken und erbielt dadurch nur eine geringe Menge von Aethylenchlorsulfocyanid, in grösster Menge aber das Disulfocyanid $\mathrm{C}_{2} \mathrm{H}_{4}(\mathrm{SCN})_{2}$. Das bei $112^{\circ}$ bis $115^{\circ}$ destillirende Oel lieferte nur Spuren oder gar kein Chlorsulfocyanid und in grösster Menge das Disulfocyanid, von dem ersteres nicht getrennt werden konnte. Trotz der obigen Thatsache konnte ich nicht glauben, dass dieses nur ca. $4^{\circ}$ böher, als jenes früher angewandte Chlorbromid, siedende Product einen so bedentenden Einfluss auf die Bildung der in Frage kommenden Substanz haben sollte. Jedoch eine grosse Zahl von Versuchen, bei denen ich absoluten Alkohol zur Anwendung brachte, zeigte mir, dass dieses in 
der That der Fall sei, weil das nach der Simpson'schen Methode in geringer Menge erhaltene und bei $107^{\circ}-108^{\circ}$ siedende Chlorbromid das Chlorsulfocyanid in ebenso grosser Menge, als das nach Lössner dargestellte, entstehen liess. Dieser letztere Versuch überzeugte mich, dass keine isomere Verbindung gebildet wird, sondern dass wahrscheinlich das höher siedende Chlorbromid Aethylendibromid beigemengt enthält. Die bei der Kohlenstoff- und Wasserstoff-Bestimmung des höher siedenden Aethylenchlorbromids gefundenen Zahlen, obgleich dieselben nicht sehr von den für $\mathrm{C}_{2} \mathrm{H}_{4} \mathrm{ClBr}$ berechneten abwichen, liessen dennoch vermuthen, dass dieses der Fall sei, da weniger Kohlenstoff und Wasserstoff gefunden wurde. Um diese Vermuthung zu bestätigen, liess ich auf $200 \mathrm{Grm}$. Aethylendibromid weniger als die berechnete Menge von Antimonpentachlorid (nur 130 Grm., ber. 159) einwirken, um so Aethylendibromid überschüssig zu haben. Ich erhielt ein Chlorbromid, welches höher als reines, nämlich von $109^{\prime \prime}-11^{\circ}$ "siedete, und aus welchem nur kleine Mengen von Aethylenchlorsulfocyanid dargestellt werden konnten.

Auch schien mir die Temperatur, bei der Chlor in die Flüssigkeit geleitet wird, einen Einfluss auf den Siedepunkt und die Ausbeute des Chlorbromids zu haben. In Folge dessen führte ich eimen neuen Versuch aus und brachte 200 Grm. Brom mit 24 ,Fluid ounces" Salzsäure und Wasser zu gleichen Volumen in einen Kolben zusammen, umgab diesen mit kleinen Stückchen Eis und liess ihn eine halbe Stunde stehen, ehe ich Chlor einleitete. Die Temperatur der Flüssigkeit betrug dann $2^{0}$.

Unter häufigem Durchschütteln wurde jetzt erst mit dem Einleiten des Chlors begonnen, und diese Operation so lange fortgesetzt bis alles ungelöste Brom verschwunden und Sättigung eingetreten war. Das nach dem Einleiten von Aethylen gebildete Oel, welches sich zu Boden gesetzt hatte, wurde nach dem Waschen mit alkalischem Wasser getrocknet und destillirt. Auf diese Weise wurden aus $200 \mathrm{Grm}$. Brom $140 \mathrm{Grm}$. des bei $107^{\circ}-109^{\circ}$ siedenden (also reinen) Aethylenchlorbromids erhalten. Andere Versuche haben eine noch grössere Ausbente ergeben, aber nicht mehr als $70 \mathrm{pCt}$. der berechneten Menge. Die Quantität von Aethylenchlorsulfocyanid, welche aus dem so erhaltenen Product gewonnen wurde, war dieselbe wie die aus dem nach der Lössner'schen Methode dargestellten Chlorbromid, obgleich obige Verbindung etwas höher siedete.

Wendet man sehr viel fein gestossenes Eis an und lässt 
und Abkömmlinge des Aethylenchlorsulfocyanids. 381

den Kolben in demselben so lange stehen bis die Temperatur der Flüssigkeit fast bis $0^{\circ}$ gesunken ist, ehe man Chlor einleitet, so wird es möglich, grössere Mengen von Brom, etwa 500 Grm., zur Anwendung zu bringen. Das so gebildete Aethylenchlorbromid destillirt zum grössten Theil bei $107^{\circ}-109^{\circ}$; doch ist die Ausbeute eine kleinere; es scheint daher vortheilhafter, mit geringeren Mengen von Brom zu operiren. Als rathsam erwies es sich ferner, das Einleitungsrohr in das Brom eintauchen zu lassen, da letzteres dann viel schneller verschwindet.

Aus obigen Versuchen ist zu folgern, dass die Temperatur, bei der das Chlor eingeleitet wird, von grosser Wichtigkeit sowohl für eine gute Ausbeute, als auch für ein zur weiteren Verarbeitung brauchbares Product ist.

Verhalten des Aethylenchlorsulfocyanids. ${ }^{1}$ )

Alkoholisches Ammoniak wirkt auf Aethylenchlorsulfocyanid bei $100^{\circ}$ unter Abscheidung von Chlorammonium ein; das in Lösung enthaltene Produkt ist ein unkrystallisirbarer, nicht unzersetzt destillirender Syrup.

Einwirkung von schwefligsaurem Natron auf Aethylenchlorsulfocyanid. Bildung von Sulfocyanäthylsulfonsäure $\mathrm{O}_{2} \mathrm{H}_{4}(\mathrm{SCN}), \mathrm{SO}_{2} \mathrm{OH}$.

Das Natriumsalz dieser neuen Säure wird mit mehr oder weniger Natriumchlorid und -sulfat vermischt erhalten durch Erwärmen des Aethylenchlorsulfocyanids mit einer wässrigen Lösung von neutralem schwefligsaurem Natron auf dem Sandbade. Besser ist folgendes Verfahren:

20 Grm. Aethylenchlorsulfocyanid wurden in einen Stöpselcylinder mit einer nicht $\mathrm{zu}$ concentrirten, wässrigen Lösung von $20 \mathrm{Grm}$. krystallisirtem schwefligsaurem Natron zusammengebracht. Die Flüssigkeit färbt sich sofort rosenroth und nimmt in kaum einer Stunde eine carminrothe Farbe an. Der Cylinder wurde nun unter häufigem Durchschütteln dem directen Sonnenlicht ausgesetzt; während eines Zeitraums von einem Tage war alles Oel unter Bildung eines braunen amorphen Körpers verschwunden, den ich nicht näher untersucht habe. Die dunkelbraune Flüssigkeit wurde filtrirt, mit ausgeglühter Thierkohle geschüttelt und abermals filtrirt; sie war jetzt fust farblos geworden. Dann wurde dieselbe unter Hinzufügung von wenig verdünnter Salzsäure auf dem Wasserbade zur Trockene gebracht, um noch unzersetztes Natriumsulfit zu zerstören, in Wasser gelöst und ein

') Vergl. dies. Journ. [2] 20, 352. 
Theil ron Chlornatrium und schwefelsaurem Natron durch Krystallisation entfernt.

Eine Analyse des Rückstandes zeigte zur Genüge, dass das Natriumsalz einer neuen Säure in demselben zu ca. $60 \mathrm{pCt}$. vorhanden war, dessen Bildung durch die folgende Gleichung ausgedrückt wird:

$\mathrm{C}_{2} \mathrm{H}_{4} \mathrm{ClSCN}+\mathrm{Na}_{2} \mathrm{SO}_{3}=\mathrm{C}_{2} \mathrm{H}_{4} \mathrm{SCNSO}_{3} \mathrm{Na}+\mathrm{NaCl}$.

Eine Trennung des gebildeten Salzes von den unorganischen Beimengungen durch Alkohol war nicht möglich, da ersteres in demselben unlöslich ist.

Dic oben erwähnte Analyse des bei $100^{\circ}$ getrockneten Rückstandes ergab $\mathrm{NaCl}, \mathrm{Na}_{2} \mathrm{SO}_{4}$ und ein organisches Salz.

Der Mittelwerth von zwei Chlorbestimmungen lieferte 24,35\% $\mathrm{NaCl}$, und von zwei Schwefelsüurebestimmungen $16,00^{\circ}{ }_{10} \mathrm{Na}_{3} \mathrm{SO}_{4}$; es berechnen sich daraus $40,35 \%$ Verumreinigungen. Resultate:

Eine Stickstoffbestinumung des unreinen salzes ergab folgende

I. $0,226 \mathrm{Grm}$. der bei $100^{\circ}$ getrockneten Substanz lieferten nach Will-Varrentrapp'schen Methode 0,0745 Grm. Platin, entsprechend $0,01058 \mathrm{Grm}$. Stickstoff.

II. 0,3357 Grm. Substanz gaben, in derselben Weise behandelt, $0,1060 \mathrm{Grm}$. Platin, entsprechend $0,0150 \mathrm{Grm}$. Stickstoff.

Diese Werthe ergeben nach Abzug der zu 40,35\% gefundenen Verunreinigungen Zahlen, welche sehr nahe mit den berechneten Werthen des Stickstoffs in der Verbindung $\mathrm{C}_{2} \mathrm{H}_{4} \mathrm{SCN} \mathrm{SO}_{3} \mathrm{Na}$ übereinstimmen.

$0,226-0,0912\left(=\mathrm{NaCl}+\mathrm{Na}_{2} \mathrm{SO}_{4}\right)=0,1348$ Grm. reines Salz.

$0,3357-0,1351(=\quad " \quad$ " $=0,2003 \quad " \quad, \quad$,

Berechnet für $\mathrm{C}_{2} \mathrm{H}_{4}<\mathrm{SO}_{3} \mathrm{Na} \quad$ Gefunden.

$$
\mathrm{N} \quad 7,40 \% \quad 7,84 \quad 7,48
$$

Die Salzmischung zieht an der Luft sehr stark Wasser an und verwandelt sich mit concentrirter Salpetersäure unter heftiger Einwirkung wahrscheinlich in ein saures Salz der Aethylendisulfonsäure: $\mathrm{C}_{2} \mathrm{H}_{4} \backslash \mathrm{SO}_{3} \mathrm{Ha}$

\section{Salze der $\beta$-Chloräthylsulfonsäure.}

Die Leichtigkeit, mit der die $\beta$-Chloräthylsulfonsäure aus dem Aethylenchlor'sulfocyanid ${ }^{1}$ ) erhalten werden kann, veranlasste mich, einige ihrer Salze und das Chlorid darzustellen.

Die Salze gewinnt man durch Behandeln der $\beta$-Chloräthylsulfonsäure mit den kohlensauren Salzen der Metalle, resp. durch Zersetzung von $\beta$-chloräthylsulfonsaurem Baryt mit schwefelsauren Salzen.

Das Kalisalz krystallisirt aus der concentrirten wässrigen Lösung in zarten, wasserfreien Nadeln. - Das Baryt-

1) Vergl. dies. Journ. [2] 20, 354. 
und Abkömmlinge des Aethylenchlorsulfocyanids. 383

salz: $\left(\mathrm{C}_{2} \mathrm{H}_{4} \mathrm{ClSO}_{2}\right)_{2} \mathrm{O}_{2} \mathrm{Ba}+2 \mathrm{H}_{2} \mathrm{O}$ wurde früher (a. a. $\mathrm{O}$. S. 354) beschrieben.

Das Strontiumsalz, $\left(\mathrm{C}_{2} \mathrm{H}_{4} \mathrm{ClSO}_{3}\right)_{2} \mathrm{Sr}+2 \mathrm{H}_{2} \mathrm{O}$, kann leicht dadurch hergestellt werden, dass man eine wässrige Lösung der Säure mit einem Ueberschuss von in Wasser suspendirtem Strontiumcarbonat versetzt. Dasselbe krystallisirt in Nadeln, welche $2 \mathrm{Mol}$. Wasser enthalten. Die Analyse ergab folgende Zahlen:

I. 0,1896 Grm. des zwischen Fliesspapier getrockneten Salzes verloren bei $100^{\circ} 0,017 \mathrm{Grm}$. Wasser.

II. 0,2842 Grm. gaben nach dem Erhitzen bis zur Rothgluth und Behandeln mit S'chwefelsäure 0,1252 Grm. $\mathrm{SrSO}_{4}$, entsprechend 0,0596 Grm. Strontium.

$$
\begin{array}{ccc} 
& \text { Berechnet. } & \text { Gefunden. } \\
\mathrm{Sr} & 21,29 \% & 21,00 \% \\
2 \mathrm{H}_{2} \mathrm{O} & 8,76 \% & 8,96 \%
\end{array}
$$

Das Zinksalz, $\left(\mathrm{C}_{2} \mathrm{H}_{4} \mathrm{ClSO}_{3}\right)_{2} \mathrm{Zn}+6 \mathrm{H}_{2} \mathrm{O}$, krystallisirt in Blättchen und ist in absolutem Alkohol und Aether unlöslich, löslich dagegen in verdünntem Alkohol.

$0,094 \mathrm{Grm}$. des lufttrocknen Salzes verloren bei $100^{\circ} 0,022 \mathrm{Grm}$. Wasser $=23,40 \%$, berechnet für $6 \mathrm{H}_{3} \mathrm{O} 23,47 \%$.

Das $\mathrm{Kupfersalz,}\left(\mathrm{C}_{2} \mathrm{H}_{4} \mathrm{ClSO}_{3}\right)_{2} \mathrm{Cu}+4 \mathrm{H}_{2} \mathrm{O}$, gewinnt man in schönen, viereckigen, blauen Tafeln des triklinen Systems, welche in Wasser leicht, in Alkohol nicht löslich sind. Von seinen vier Molekülen Krystallwasser gehen zwei unter Hellerwerden der Farbe bei $100^{\circ}$ fort, die übrigen erst zwischen $120^{\circ}-125^{\circ}$. Das Salz sieht dann weiss aus. Höher als $125^{\circ}$ erleidet es geringe Zersetzung.

I. $0,338 \mathrm{Grm}$. Salz verloren bei $100^{\circ} 0,032 \mathrm{Grm}$., und beim Erhitzen auf $125^{\circ}$ betrug der Gesammtverlust $0,0575 \mathrm{Grm}$.

II. $0,3015 \mathrm{Grm}$. verloren bei $100^{\circ} 0,0280 \mathrm{Grm}$. und bei $125^{\circ} 0,048 \mathrm{Grm}$.

$$
\begin{array}{cccc}
\text { Berechnet } & \multicolumn{3}{c}{\text { Gefunden }} \\
\left(\mathrm{C}_{2} \mathrm{H}_{4} \mathrm{ClSO}_{8}\right)_{2} \mathrm{Cu}+4 \mathrm{H}_{2} \mathrm{O} . & \text { bei } 100^{\circ} \text {. bei } 125^{\circ} . \\
2 \mathrm{H}_{2} \mathrm{O} \quad 8,53 \% ; \quad 4 \mathrm{H}_{2} \mathrm{O} \quad 17,06 \% & \text { I. } 9,46 & 16,86 \\
& \text { II. } 9,28 & 16,08
\end{array}
$$

$\beta$-Chloräthylsulfonsäure-Chlorid, $\mathrm{C}_{2} \mathrm{H}_{4} \mathrm{ClSO} \mathrm{Sl}_{2} \mathrm{Cl}$ durch die Einwirkung der berechneten Menge von FünffachChlorphosphor auf $50 \mathrm{Grm}$. chloräthylsulfonsaures Kali dargestellt und durch Destillation gereinigt, siedet bei $200^{\circ}$ bis $203^{\circ}$ (uncorr.).

Durch Einwirkung von Ammoniakgas auf das in Aether gelöste Chlorid entsteht unter Abscheidung von Chlorammonium ein chlorfreies, stickstoff- und schwefelhaltiges Oel, dessen Zusammensetzung noch nicht ermittelt ist. 
Da durch obige Reaction wider Erwarten nicht das Amid der Chloräthylsulfonsäure entsteht, so wurde der Versuch gemacht, aus A ethylsulfonsäurechlorid mittelst Ammoniak das Amid der Aethylsulfonsäure darzustellen, was ohne Schwierigkeit gelang. Dasselbe krystallisirt aus der ätherischen Lösung in langen glänzenden, bei $58^{\circ}$ schmelzenden Prismen, löst sich in Wasser, Alkohol, Aether.

$0,4080 \mathrm{Grm}$. gaben $48 \mathrm{Ccm}$. Stickstoff bei $26^{\circ}$ und $754 \mathrm{Mm}$. Barometerstand, entsprechend $12,86 \%$; das Amid enthält $12,84 \% \mathrm{~N}$.

Bristol, October 1882.

\section{Vorläufige Mittheilung;}

von

\section{Dr. Wilh. Ostwald,}

Professor am Polytechnicum zu Riga.

Veranlasst durch die im letzten Hefte der Berichte der. Berl, chem. Ges, enthaltenen Notizen von Menschutkin und L. Meyer, Untersuchungen über die Bildung und Zerlegung der Amide, resp. Anilide betreffend, theile ich mit, dass ich seit einem halben Jahre mich mit ähnlichen Arbeiten über die Einwirkung von Säuren auf Amide, speciell Acetamid, beschäftige. Es bildet sich, wie bekannt, bei dieser Einwirkung Ammoniaksalz; die Geschwindigkeit der Umwandlung ist von der Natur der Säure abhängig und steht in einer sehr einfachen Beziehung zu der Grösse, welche ich die relative Affinität genannt habe; sie ist nahezu proportional dem Quadrat derselben.

Die analytische Methode zur Bestimmung des Ammoniaksalzes neben unzersetztem Amid besteht in der Behandlung mit unterbromigsaurem Natron im Azotometer, durch welche das Amid keine Veränderung erleidet, während der Stickstoff des Ammoniaks momentan entwickelt wird.

Ich bin auf diesem Wege zu einer Messung von Affinitätsgrössen durch die entsprechenden Geschwindigkeiten gelangt, während die bisher benutzten Methoden chemisches Gleichgewicht, d. h. die Geschwindigkeit Null voraussetzten. An die bisher fast ausschliesslich gepflegte chemische Statik schliessen sich hier in consequenter Entwickelung die Anfänge einer chemischen Dynamik an.

Die ausführliche Mittheilung meiner Versuchsergebnisse hoffe ich in kurzer Frist machen zu können.

Riga, October 1882. 